\title{
Application of a Modified Homotopy Perturbation Method for Calculation of Secular Axial Frequencies in a Nonlinear Ion Trap with Hexapole, Octopole and Decapole Superpositions
}

\section{Alireza Doroudi*}

Physics Department, Nuclear Science Research School, Nuclear Science and Technology Research Institute (NSTRI), P.O. Box 14395-836, Tehran, Iran

\begin{abstract}
In this paper we have used a modified homotopy perturbation method used previously by A. Belendez and his coworkers, for calculation of axial secular frequencies of a nonlinear ion trap with hexapole, octopole and decapole superpositions. We transform the motion of the ion in a rapidly oscillating field to the motion in an effective potential and obtain a nonlinear differential equation in the form of a Duffing-like equation. With only octopole superposition the resulted nonlinear equations are symmetric; however, in the presence of hexapole and decapole superpositions they are asymmetric. For asymmetric oscillators, it has been pointed out that the angular frequency for positive amplitudes is different from the angular frequency for negative amplitudes. Considering this problem, the modified homotopy perturbation method is used for solving the resulted nonlinear equations. As a result, the ion axial secular frequencies as a function of nonlinear field parameters are obtained. The calculated secular frequencies are compared with the results of modified Lindstedt-Poincare approximation and the exact results. There is an excellent agreement between the results of this paper and the exact results.
\end{abstract}

Keywords: Modified homotopy perturbation; Nonlinear ion trap; Secular frequency; Hexapole; Octopole; Decapole; Symmetric oscillator; Asymmetric oscillator

\section{Introduction}

In an ideal ion trap the potential is pure quadrupole and the main properties of the movement of an ion are obtained by the solution of Mathieu equation [1]. In a practical ion trap, however, the electric field distribution deviates from linearity which is the characteristic of pure quadrupolar trap geometry. This deviation is caused by many different agents such as the truncation of electrodes.

The nonlinearity in ion traps superimpose weak multipole fields (e.g., hexapole, octopole, decapole, and higher order fields) and the resulting nonlinear field ion traps exhibit some effects which differ considerably from those of the linear field traps.

The equation governing the motion of the ion in the nonlinear ion trap is the nonlinear Mathieu equation which cannot be solved analytically. The superposition of weak higher multipole fields changes the motions of ions compared to their motions in a pure quadrupole ion trap.

Simulation studies [2] have shown that hexapole superposition decreases the secular frequency, positive octopole superposition increases the ion secular frequency and the negative octopole superposition decreases the secular frequency. Experimentally, it has been shown that [3] the octopole and hexapole superposition resulted in a decrease in ion secular frequency.

Sevugarajan and Menon [4-6] have studied the nonlinear Paul ion trap. They have applied the Lindstedt-Poincare technique, the modified Lindstedt-Poincare technique and the multiple scales perturbation technique for solving the nonlinear equation of ion motion in nonlinear ion trap. Also, in two previous studies $[7,8]$ done on nonlinear ion traps by one of the present authors, the homotopy perturbation method was used to study the secular frequencies in nonlinear ion traps. When the hexapole superposition is considered, the resulting nonlinear equation has a quadratic nonlinearity and we know that the angular frequency for positive amplitudes is different from the angular frequency for negative amplitudes in nonlinear oscillator with quadratic nonlinearity. In all the above studies [4-8] the assumption is that the angular frequency for positive amplitudes is equal to the angular frequency for negative amplitudes.

In studying the quadratic nonlinear oscillator and mixed parity nonlinear oscillator by the method of harmonic balance, $\mathrm{H}$. $\mathrm{Hu}$ $[9,10]$ has used the sign function for incorporating the inequality of angular frequency for negative amplitudes and positive amplitudes. In a recent study [11], this technique was used in the framework of the parameter expanding or modified Lindstedt-Poincare method [12-14] for calculation of secular axial frequencies in a nonlinear ion trap with hexapole, octopole and decapole superpositions. In this paper, we use the same technique and the homotopy perturbation method [14-25] with a modification [26-28] for studying the asymmetric nonlinear oscillators. The modification in He's homotopy perturbation method is introduced by truncating the infinite series corresponding to the first order approximate solution before introducing this solution in the second order linear differential equation and so on.

The exact solution for nonlinear equation of an anharmonic oscillator with quadratic nonlinearity and the exact expression for its period have been studied by some authors $[29,30]$. They have found the exact expression for the period of nonlinear oscillator in terms of complete elliptic integrals. We have used the results of these papers and

*Corresponding author: Amineh Rezaeian Asl, Department of Physics, Faculty of Science, Islamic Azad University, Central Tehran Branch, Tehran, Iran, E-mail: Adoroudi@aeoi.org.ir

Received September 09, 2012; Accepted October 17, 2012; Published October 22, 2012

Citation: Doroudi A (2012) Application of a Modified Homotopy Perturbation Method for Calculation of Secular Axial Frequencies in a Nonlinear Ion Trap with Hexapole, Octopole and Decapole Superpositions. J Bioanal Biomed 4: 085-091. doi:10.4172/1948-593X.1000068

Copyright: (C) 2012 Doroudi A. This is an open-access article distributed under the terms of the Creative Commons Attribution License, which permits unrestricted use, distribution, and reproduction in any medium, provided the original author and source are credited. 
have calculated the exact frequencies of an anharmonic oscillator with quadratic nonlinearity. The mathematica software has been used for calculation of elliptic integrals.

In this paper we take into account the first four multipole terms of potential distribution inside the ion trap, i.e. quadrapole, hexapole, octopole and decapole terms and ignore the higher multipole fields. The resulting nonlinear equation has quadratic and cubic as well as quartic nonlinearity. Due to the inequality of angular frequency for negative amplitudes and positive amplitudes, we use the sign function for constructing the two auxiliary nonlinear equations. Then, the modified homotopy perturbation method [26-28] is used for solving the two auxiliary nonlinear differential equations and the ion secular frequencies are calculated. We compare the results of this paper with those obtained by using the modified Lindstedt-Poincare method [11] and with the exact results.

The outline of the paper is as follows: In section 2 the axial equation of ion motion in a nonlinear ion trap is derived. In section 3 the modified homotopy perturbation method is applied to solve the equation of ion motion in nonlinear ion trap and the results are also given in this equation. Finally, the concluding remarks are given in section 4 .

\section{The Axial Equation of Ion Motion in a Nonlinear Ion Trap}

The axial equation of ion motion in the presence of hexapole and octopole superpositions has been derived in $[7,8]$ and in the presence of hexapole, octopole, and decapole superposition has been derived in [11]. However, in this section, we give a brief derivation of the axial equation of ion motion in the latter case.

A solution of Laplace's equation in spherical polar coordinates for a system with axial symmetry can be written in the following general form [31]:

$$
\varphi(\rho, \vartheta, \phi)=\varphi_{0} \sum_{n=0}^{\infty} A_{n} \frac{\rho^{n}}{r_{0}^{n}} P_{n}(\cos \vartheta)
$$

where $\varphi_{0}=U+V \cos \Omega t$ is the potential applied to the trap, $A_{n}$ 's are arbitrary dimensionless coefficients, $P_{n}(\cos \vartheta)$ denotes a Legendre polynomial of order $n$, and $r_{0}$ is a scaling factor (i.e., the internal radius of the ring electrode).

When $\rho^{n} P_{n}(\cos \vartheta)$ is expressed in cylindrical polar coordinates $(r, z)$ and the three higher order multipoles, i.e. hexapole, octopole and decapole corresponding to $n=3,4$ and 5 along with the quadrupole component corresponding to $n=2$ are taken into account, the time dependent potential distribution inside the trap takes the form:

$$
\begin{aligned}
& \varphi(r, z, t)=\frac{A_{2}}{r_{0}^{2}} V \cos \Omega t \\
& {\left[\begin{array}{l}
\frac{2 z^{2}-r^{2}}{2}+\frac{f_{1}}{r_{0}}\left(\frac{2 z^{3}-3 r^{2} z}{2}\right)+ \\
\frac{f_{2}}{r_{0}{ }^{2}}\left(\frac{8 z^{4}-24 z^{2} r^{2}+3 r^{4}}{8}\right)+\frac{f_{3}}{r_{0}{ }^{3}}\left(\frac{8 z^{5}-40 z^{3} r^{2}+15 z r^{4}}{8}\right)
\end{array}\right]}
\end{aligned}
$$

where $f_{1}=A_{3} / A_{2}, f_{2}=A_{4} / A_{2}$ and $f_{3}=A_{5} / A_{2}$. Here we have assumed the operation of the trap along the $a_{u}=0$ axis in the Mathieu stability plot, that is, the DC component of $\varphi_{0}$ is equal to zero. The coefficients $A_{2}, A_{3}, A_{4}$ and $A_{5}$ refer to the weight of the quadrupole, hexapole, octopole and decapole superpositions, respectively.

According to classical mechanics [32], the motion of an ion in a rapidly oscillating field such as $\varphi(r, z, t)$ (due to the largeness of $\Omega$ ) can be averaged and transformed to the motion in an effective potential, $U_{\text {eff }}(r, z)$, related to $\varphi(r, z, t)$ through the following relation:

$$
U_{e f f}(r, z)=\frac{e}{2 m}\left\langle\left|\int \vec{\nabla} \varphi(r, z, t) d t\right|^{2}\right\rangle
$$

Insertion of Eq. (2) for $\varphi(r, z, t)$ into Equation. (3) and averaging with respect to time gives the following relation for $U_{\text {eff }}(r, z)$,

$$
\begin{aligned}
& U_{e f f}(r, z)=\frac{1}{\lambda} \omega_{0 u}^{2}\left(\frac{m}{e}\right)\left[r^{2}+4 z^{2}+\frac{f_{1}^{2}}{r_{0}^{2}}\left(9 z^{4}+\frac{9}{4} r^{4}\right)+\frac{12 f_{1}}{r_{0}} z^{3}\right. \\
& \left.+\frac{f_{2}}{r_{0}^{2}}\left(16 z^{4}-3 r^{4}-12 r^{2} z^{2}\right)+\frac{f_{3}}{r_{0}^{3}}\left(20 z^{5}-40 r^{2} z^{3}-\frac{15}{2} r^{4} z\right)\right]
\end{aligned}
$$

where $\lambda=2$ for $u=r$ (radial direction) and $\lambda=8$ for $u=z$ (axial direction).

By ignoring the term proportional to $f_{1}^{2}$ compared with the term proportional to $f_{1}$ (because $f_{1}=A_{3} / A_{2}$ is small in comparison to 1 ), the final form of $U_{\text {eff }}(r, z)$ reduces to the following form,

$$
\begin{aligned}
& U_{\text {eff }}(r, z)=\frac{1}{\lambda} \omega_{0 u}^{2}\left(\frac{m}{e}\right)\left[r^{2}+4 z^{2}+\frac{12 f_{1}}{r_{0}} z^{3}\right. \\
& \left.+\frac{f_{2}}{r_{0}^{2}}\left(16 z^{4}-3 r^{4}-12 r^{2} z^{2}\right)+\frac{f_{3}}{r_{0}^{3}}\left(20 z^{5}-40 r^{2} z^{3}-\frac{15}{2} r^{4} z\right)\right]
\end{aligned}
$$

The classical equation of ion motion in the effective potential $U_{\text {eff }}(r, z)$, and with no excitation potential applied to the end cap electrodes is given by:

$$
\frac{d^{2} \vec{r}}{d t^{2}}+\frac{e}{m} \vec{\nabla} U_{e f f}(r, z)=0
$$

Where $\vec{r}$ is the position vector of the ion. Combining equations (5) and (6), we get the equation of motion in the axial $(z)$ direction as:

$$
\frac{d^{2} z}{d t^{2}}+\omega_{0 z}^{2} z+\alpha_{2}^{\prime} z^{2}+\alpha_{3}^{\prime} z^{3}+\alpha_{4}^{\prime} z^{4}+\alpha_{5}^{\prime} r^{2} z+\alpha_{6}^{\prime} r^{2} z^{2}+\alpha_{7}^{\prime} r^{4}=0
$$

This is the equation in $z$ direction which is coupled to equation in $r$ direction. Since we are interested in axial secular frequencies, we put $r=0$ in equation (7) and get an equation in axial direction which depends only on $z$ variable:

$$
\frac{d^{2} z}{d t^{2}}+\omega_{0 z}^{2} z+\alpha_{2}^{\prime} z^{2}+\alpha_{3}^{\prime} z^{3}+\alpha_{4}^{\prime} z^{4}=0
$$

where

$$
\begin{aligned}
& \omega_{0 z}=\frac{q_{z} \Omega}{2 \sqrt{2}} \\
& q_{z}=\frac{4 e V}{m r_{0}^{2} \Omega^{2}} \\
& \alpha_{2}^{\prime}=\frac{9 f_{1} \omega_{0 z}^{2}}{2 r_{0}}
\end{aligned}
$$




$$
\begin{aligned}
& \alpha_{3}^{\prime}=\frac{8 f_{2} \omega_{0 z}^{2}}{r_{0}^{2}} \\
& \alpha_{4}^{\prime}=\frac{25 f_{3} \omega_{0 z}^{2}}{2 r_{0}^{3}}
\end{aligned}
$$

In Equation (8), by introducing the dimensionless variable $x$ through the relation $x=z / r_{0}$, and omission of index $z$ from $\omega_{0 z}$ (for simplicity) we get the equation,

$$
\ddot{x}+\omega_{0}^{2} x+\alpha_{2} x^{2}+\alpha_{3} x^{3}+\alpha_{4} x^{4}=0,
$$

where $\alpha_{2}=(9 / 2) f_{1} \omega_{0}^{2}, \alpha_{3}=8 f_{2} \omega_{0}^{2}$ and $\alpha_{4}=(25 / 2) f_{3} \omega_{0}^{2}$.

There are several methods $[33,34]$ that can be used for solution of the nonlinear Equation (14). In the next section of this article we have used the modified homotopy perturbation method for solving this equation.

\section{Application of Modified Homotopy Perturbation Method for Solution of the Axial Equation of Motion and the Results}

The homotopy perturbation method [14-25] is a general method for solving the differential and integral equations. This method is explained in references 7 and 8 for solving a nonlinear differential equation like Equation (14). In this method an embedding parameter $p \in[0,1]$ is introduced. The periodic solution of the nonlinear differential equation and the coefficient of linear term $\left(\omega_{0}^{2}\right)$ are assumed to be written as power series in $p$. Then, the power series for the solution and $\omega_{0}^{2}$ are inserted in the nonlinear equation and a set of linear differential equations is obtained for the coefficients of the power series for the periodic solution. The first few linear equations of the set are solved and then are inserted in the power series along with $p=1$. In this manner, an approximate solution of the main nonlinear differential equation is obtained. Here we use this method for solving the equation (14).

The nonlinear differential equation $\ddot{x}+\omega_{0}^{2} x+\alpha_{2} x^{2}+\alpha_{3} x^{3}$ $+\alpha_{4} x^{4}=0$ is the equation of a mixed parity nonlinear oscillator and the amplitudes of oscillations for this oscillator are not the same when $x \geq 0$ and $x \leq 0$. We assume that the positive amplitude is $A$ and the negative amplitude is $-B$ ( $B$ is positive). Now we construct the two auxiliary equations by using sign function:

$$
\begin{aligned}
& \ddot{x}+\omega_{0}^{2} x+\alpha_{2} x^{2} \operatorname{sgn}(x)+\alpha_{3} x^{3}+\alpha_{4} x^{4} \\
& \operatorname{sgn}(x)=0 \quad x \geq 0, x(0)=A, \quad \dot{x}(0)=0 \\
& \ddot{x}+\omega_{0}^{2} x-\alpha_{2} x^{2} \operatorname{sgn}(x)+\alpha_{3} x^{3}-\alpha_{4} x^{4} \\
& \operatorname{sgn}(x)=0 \quad x \leq 0, x(0)=-B \quad \dot{x}(0)=0
\end{aligned}
$$

The sign function is defined as:

$$
\operatorname{sgn}(x)= \begin{cases}1, & x>0 \\ 0, & x=0(17) \\ -1, & x<0\end{cases}
$$

First, we consider the Equation (15) and construct the following homotopy:

$$
\begin{aligned}
& \ddot{x}+\omega_{0}^{2} x+p\left[\alpha_{2} x^{2} \operatorname{sgn}(x)+\alpha_{3} x^{3}+\alpha_{4} x^{4}\right. \\
& \operatorname{sgn}(x)]=0 \quad x(0)=A, \quad \dot{x}(0)=0
\end{aligned}
$$

In homotopy perturbation method the solution $x$ and the constant $\omega_{0}^{2}$ are expanded in powers of embedding parameter $p$ as:

$$
\begin{aligned}
& x=x_{0}+p x_{1}+p^{2} x_{2}+\cdots \cdots \\
& \omega_{0}^{2}=\omega_{A}^{2}+p \omega_{A 1}+p^{2} \omega_{A 2}+\cdots \cdots
\end{aligned}
$$

In equation (15), the amplitude is $A$, so we have used the index $A$ in the expansion of $\omega_{0}^{2}$. As a result, in the expansion of $\omega_{0}^{2}$ for solution of equation (16) we use the index $B$.

Substitution of the power series (19) and (20) into Equation (18), and collecting terms of the same power of $p$, gives the following set of linear equations:

$$
\begin{aligned}
& \ddot{x}_{0}+\omega_{A}^{2} x_{0}=0, x_{0}(0)=A \quad \dot{x}_{0}(0)=0 \\
& \ddot{x}_{1}+\omega_{A}^{2} x_{1}+\omega_{A 1} x_{0}+\alpha_{2} x_{0}^{2} \operatorname{sgn}\left(x_{0}\right)+\alpha_{3} x_{0}^{3}+\alpha_{4} x_{0}^{4} \\
& \operatorname{sgn}\left(x_{0}\right)=0 \quad x_{1}(0)=0 \quad \dot{x}_{1}(0)=0 \\
& \ddot{x}_{2}+\omega_{A}^{2} x_{2}+\omega_{A 2} x_{0}+\omega_{A 1} x_{1}+2 \alpha_{2} x_{0} x_{1} \operatorname{sgn}\left(x_{0}\right) \\
& +3 \alpha_{3} x_{0}^{2} x_{1}+4 \alpha_{4} x_{0}^{3} x_{1} \operatorname{sgn}\left(x_{0}\right)=0 \quad x_{2}(0)=0 \quad \dot{x}_{2}(0)=0
\end{aligned}
$$

In Equation (21)-(23) we have taken into account the following expression $[35,36]$,

$$
\begin{aligned}
& f(x)=f\left(x_{0}+p x_{1}+p^{2} x_{2}+\cdots\right)=f\left(x_{0}\right)+p x_{1} f^{\prime}\left(x_{0}\right)+ \\
& p^{2}\left[x_{2} f^{\prime}\left(x_{0}\right)+\frac{1}{2} x_{1}^{2} f^{\prime \prime}\left(x_{0}\right)\right]+\mathrm{O}\left(p^{3}\right)
\end{aligned}
$$

where $f^{\prime}(x)=d f(x) / d x$ and $d \operatorname{sgn}(x) / d x=d^{2} \operatorname{sgn}(x) / d x^{2}=\cdots=0$ for $x \neq 0$ and $\operatorname{sgn}\left(x_{0}+p x_{1}+p^{2} x_{2}+\cdots\right)=\operatorname{sgn}\left(x_{0}\right)$

The first equation of the set can be solved easily, giving the solution $x_{0}(t)=A \cos \omega_{A} t$. we can expand the terms $x_{0}^{2} \operatorname{sgn}\left(x_{0}\right)$ and $x_{0}^{4} \operatorname{sgn}\left(x_{0}\right)$ in Equation (22) in Fourier series as:

$$
\begin{aligned}
& x_{0}^{2} \operatorname{sgn}\left(x_{0}\right)=A^{2} \sum_{n=0}^{\infty} a_{2 n+1} \cos \left[(2 n+1) \omega_{A} t\right]= \\
& 8 A^{2}\left[\frac{1}{3 \pi} \cos \omega_{A} t+\frac{1}{15 \pi} \cos 3 \omega_{A} t-\frac{1}{105 \pi} \cos 5 \omega_{A} t+\cdots \cdots \cdot\right]
\end{aligned}
$$

Where,

$$
a_{2 n+1}=\frac{8(-1)^{n}}{\left(3+2 n-12 n^{2}-8 n^{3}\right) \pi}
$$

and

$$
\begin{aligned}
& x_{0}^{4} \operatorname{sgn}\left(x_{0}\right)=A^{4} \sum_{n=0}^{\infty} c_{2 n+1} \cos \left[(2 n+1) \omega_{A} t\right] \\
& =32 A^{4}\left[\frac{1}{15 \pi} \cos \omega_{A} t+\frac{1}{35 \pi} \cos 3 \omega_{A} t+\frac{1}{315 \pi} \cos 5 \omega_{A} t+\cdots \cdots \cdot\right]
\end{aligned}
$$

where,

$$
c_{2 n+1}=\frac{96(-1)^{n}}{\left(45+18 n-200 n^{2}-80 n^{3}+80 n^{4}+32 n^{5}\right) \pi}
$$

Substituting $x_{0}(t)$, as well as the above series expansions for $x_{0}^{2} \operatorname{sgn}\left(x_{0}\right)$ and $x_{0}^{4} \operatorname{sgn}\left(x_{0}\right)$ into Eq. (22), having no secular term in solution $x_{1}(t)$ requires eliminating contributions proportional to $\cos \omega_{A} t$ in Eq. (22) and we obtain:

$$
\omega_{A 1}=-\frac{8 A \alpha_{2}}{3 \pi}-\frac{3 A^{2} \alpha_{3}}{4}-\frac{32 A^{3} \alpha_{4}}{15 \pi}
$$


Insertion of $\omega_{A 1}$ into Eq. (20), neglecting the terms proportional to $p^{2}$ and higher degrees, and combining with $p=1$ at last step, the approximate amplitude dependent frequency, $\omega_{A}^{(1)}$, in first order is obtained as:

$$
\omega_{A}=\omega_{A}^{(1)}=\sqrt{\omega_{0}^{2}+\frac{8 A \alpha_{2}}{3 \pi}+\frac{3 A^{2} \alpha_{3}}{4}+\frac{32 A^{3} \alpha_{4}}{15 \pi}}
$$
Finally, insertion of $\alpha_{2}=\frac{9}{2} f_{1} \omega_{0}^{2}, \alpha_{3}=8 f_{2} \omega_{0}^{2}$ and $\alpha_{4}=\frac{25}{2} f_{3} \omega_{0}^{2}$
in this equation gives the result,

$$
\frac{\omega_{A}}{\omega_{0}}=\frac{\omega_{A}^{(1)}}{\omega_{0}}=\sqrt{1+\frac{12 f_{1} A}{\pi}+6 f_{2} A^{2}+\frac{80 f_{3} A^{3}}{3 \pi}}
$$

In a similar way, for oscillation of ion in negative direction, from Eq. (16) we get the following result for $\omega_{B}$, in first order of perturbation,

$$
\frac{\omega_{B}}{\omega_{0}}=\frac{\omega_{B}^{(1)}}{\omega_{0}}=\sqrt{1-\frac{12 f_{1} B}{\pi}+6 f_{2} B^{2}-\frac{80 f_{3} B^{3}}{3 \pi}}
$$

In first order of the perturbation, the results for $\omega_{A}$ and $\omega_{B}$ are the same as the results of modified Lindstedt-Poincare method [11].

Now, we go to second order approximation. In order to go to second order approximation we need to obtain the correction term $x_{1}(t)$ for the periodic solution $x_{0}(t)$. In order to obtain $x_{1}(t)$, we insert the expression for $\omega_{A 1}$ from Eq. (29) into Eq. (22) and rewrite it in the following form,

$$
\begin{aligned}
& \ddot{x}_{1}+\omega_{A}^{2} x_{1}=-\alpha_{2} A^{2} \sum_{n=1}^{\infty} a_{2 n+1} \cos \left[(2 n+1) \omega_{A} t\right]-\alpha_{3} A^{3} \sum_{n=1}^{\infty} b_{2 n+1} \cos \left[(2 n+1) \omega_{A} t\right] \\
& -\alpha_{4} A^{4} \sum_{n=1}^{\infty} c_{2 n+1} \cos \left[(2 n+1) \omega_{A} t\right] \quad x_{1}(0)=0 \quad \dot{x}_{1}(0)=0
\end{aligned}
$$

where, $b_{3}=\frac{1}{4}$ and $b_{2 n+1}=0$ for $n \geq 2$.

Now, the periodic solution to Equation (33) can be written in the following form,

$$
x_{1}(t)=\sum_{n=0}^{\infty} d_{2 n+1} \cos \left[(2 n+1) \omega_{A} t\right]
$$

Insertion of Equation (34) into Equation (33) gives the following expression for the coefficients $d_{2 n+1}$

$$
d_{2 n+1}=\frac{\alpha_{2} A^{2} a_{2 n+1}+\alpha_{3} A^{3} b_{2 n+1}+\alpha_{4} A^{4} c_{2 n+1}}{4 n(n+1) \omega_{A}^{2}}
$$

for $n \geq 1$.

Taking into account the initial condition $x_{1}(0)=0$, we obtain from Equation (34):

$$
d_{1}=-\sum_{n=0}^{\infty} d_{2 n+1}
$$

To determine the second order approximate solution we need to substitute Equation (34) into Equation (23). The new differential equation for $x_{2}(t)$ is difficult to solve because of an infinite number of harmonic terms in $x_{1}(t)$. At this stage we consider the modification in He's homotopy perturbation method [26-28] to simplify the solution procedure. In this modified version of homotopy method the infinite series expansion for $x_{1}(t)$ in Equation (34) is truncated and an approximate equation $x_{1}^{(N)}(t)$ is written as:

$$
x_{1}^{(N)}(t)=\sum_{n=0}^{N} d_{2 n+1} \cos \left[(2 n+1) \omega_{A} t\right]
$$

where

$$
d_{1}^{(N)}=-\sum_{n=1}^{N} d_{2 n+1}
$$

In the approximate expression for $x_{1}^{(N)}(t)$ we assume $N=2$ and obtain the approximate solution for $x_{1}$ as:

$$
x_{1}(t)=d_{3}\left(\cos 3 \omega_{A} t-\cos \omega_{A} t\right)+d_{5}\left(\cos 5 \omega_{A} t-\cos \omega_{A} t\right)
$$

$d_{3}$ and $d_{5}$ are calculated by using the relation (35).

Now, we substitute $x_{0}(t)$ and the approximate $x_{1}(t)$ into Equation (23) and expand the terms $2 x_{0} x_{1} \operatorname{sgn}\left(x_{0}\right)$ and $4 x_{0}^{3} x_{1} \operatorname{sgn}\left(x_{0}\right)$ of this equation in Fourier series. Having no secular term in solution $x_{2}(t)$ requires eliminating contributions proportional to $\cos \omega_{A} t$ in the resulted differential equation for $x_{2}(t)$, which implies:

$$
\begin{aligned}
& \omega_{A 2}=\frac{3232 A^{2} \alpha_{2}^{2}}{33075 \pi^{2} \omega_{A}^{2}}+\frac{3 A^{4} \alpha_{3}^{2}}{128 \omega_{A}^{2}}+\frac{100864 A^{6} \alpha_{4}^{2}}{297675 \pi^{2} \omega_{A}^{2}}+\frac{2 A^{3} \alpha_{2} \alpha_{3}}{21 \pi \omega_{A}^{2}} \\
& +\frac{35584 A^{4} \alpha_{2} \alpha_{4}}{99225 \pi^{2} \omega_{A}^{2}}+\frac{2 A^{3} \alpha_{2} \alpha_{3}}{21 \pi \omega_{A}^{2}}+\frac{8 A^{5} \alpha_{3} \alpha_{4}}{45 \pi \omega_{A}^{2}}
\end{aligned}
$$

Insertion of calculated expressions for $\omega_{A 1}$ and $\omega_{A 2}$ into Eq. (20) along with $p=1$ and using the values of $\alpha_{2}=\frac{9}{2} f_{1} \omega_{0}^{2}, \alpha_{3}=8 f_{2} \omega_{0}^{2}$ and $\alpha_{4}=\frac{25}{2} f_{3} \omega_{0}^{2}$ gives the final result for $\omega_{A}^{(2)}$, the second order approximate frequency and $T_{A 2}$, the second order approximate period, in positive direction:

$$
\frac{\omega_{A}}{\omega_{0}}=\frac{\omega_{A}^{(2)}}{\omega_{0}}=\frac{1}{3 \sqrt{210 \pi}} \sqrt{L_{A}+\sqrt{3} \sqrt{S_{A}}}, T_{A 2}=\frac{2 \pi}{\omega_{A}^{(2)}}
$$

Where,

$$
\left.L_{A}=315\left(36 A f_{1}+80 A^{3} f_{3}+3 \pi+18 \pi A^{2} f_{2}\right)\right)
$$

and

$$
\begin{aligned}
& S_{A}=16 A^{2}\left(2531817 f_{1}^{2}+10405800 A^{2} f_{1} f_{3}+\right. \\
& \left.9290000 A^{4} f_{3}^{2}\right)+37800 A\left(27 f_{1}\left(7+38 f_{2} A^{2}\right)+140\right. \\
& \left.A^{2}\left(3+14 f_{2} A^{2}\right) f_{3}\right) \pi+297675\left(1+6 f_{2} A^{2}\left(2+5 f_{2} A^{2}\right)\right) \pi^{2}
\end{aligned}
$$

In a similar way, for oscillation of ion in negative direction we get from Equation (16):

$$
\frac{\omega_{B}}{\omega_{0}}=\frac{\omega_{B}^{(2)}}{\omega_{0}}=\frac{1}{3 \sqrt{210 \pi}} \sqrt{L_{B}+\sqrt{3} \sqrt{S_{B}}}, T_{B 2}=\frac{2 \pi}{\omega_{B}^{(2)}}
$$

Where,

$$
\left.L_{B}=315\left(-36 B f_{1}-80 B^{3} f_{3}+3 \pi+18 \pi B^{2} f_{2}\right)\right)
$$

and

$$
\begin{aligned}
& S_{B}=16 B^{2}\left(2531817 f_{1}^{2}+10405800 B^{2} f_{1} f_{3}+\right. \\
& \left.9290000 B^{4} f_{3}^{2}\right)-37800 B\left(27 f_{1}\left(7+38 f_{2} B^{2}\right)+140\right. \\
& \left.B^{2}\left(3+14 f_{2} B^{2}\right) f_{3}\right) \pi+297675\left(1+6 f_{2} B^{2}\left(2+5 f_{2} B^{2}\right)\right) \pi^{2}
\end{aligned}
$$


Citation: Doroudi A (2012) Application of a Modified Homotopy Perturbation Method for Calculation of Secular Axial Frequencies in a Nonlinear lon Trap with Hexapole, Octopole and Decapole Superpositions. J Bioanal Biomed 4: 085-091. doi:10.4172/1948-593X.1000068

In the relations (42) and (43) $A$ is the positive amplitude and is equal to maximum value for $x$ and $x_{\max }$ can be obtained by using the relation $z_{0} / r_{0}=1 / \sqrt{2}$ for ion trap and inserting $z_{0}$ for $z$ in equation $x=z / r_{0}$ gives $A=1 / \sqrt{2}$. As mentioned earlier, the amplitude in negative direction, $B$, is different from $A$ and can be calculated in terms of $A$. For calculation of $B$, the both side of Equation (14) is multiplied by $\dot{x}$ and then integrated [10], giving the result,

$$
\frac{1}{2} \dot{x}^{2}+\frac{1}{2} \omega_{0}^{2} x^{2}+\frac{1}{3} \alpha_{2} x^{3}+\frac{1}{4} \alpha_{3} x^{4}+\frac{1}{5} \alpha_{4} x^{5}=C
$$

Where $C$ is the constant of integration. Insertion of initial conditions in Equation (47) gives the result,

$$
\begin{aligned}
& \frac{1}{2} \omega_{0}^{2} A^{2}+\frac{1}{3} \alpha_{2} A^{3}+\frac{1}{4} \alpha_{3} A^{4}+\frac{1}{5} \alpha_{4} A^{5}=\frac{1}{2} \omega_{0}^{2} B^{2}-\frac{1}{3} \alpha_{2} \\
& B^{3}+\frac{1}{4} \alpha_{3} B^{4}-\frac{1}{5} \alpha_{4} B^{5}
\end{aligned}
$$

Using mathematica software, this equation can be solved analytically and $B$ is calculated in terms of $A$.

It is clear that the second order approximate period is:

$$
T_{2}=\frac{T_{A 2}+T_{B 2}}{2}
$$

So, the second order approximate secular frequency is:

$$
\frac{\omega_{2}}{\omega_{0}}=\frac{2 \pi}{T_{2}}=\frac{2}{3 \sqrt{210 \pi}}\left(\frac{1}{\sqrt{L_{A}+\sqrt{3} \sqrt{S_{A}}}}+\frac{1}{\sqrt{L_{B}+\sqrt{3} \sqrt{S_{B}}}}\right)^{-1}
$$

The perturbed secular frequencies can be calculated through the relation (50) as a function of field aberrations (parameters $f_{1}, f_{2}$ and $\left.f_{3}\right)$.
The values of $\omega / \omega_{0}$ for different values of $f_{1}, f_{2}$ and $f_{3}$ are given in tables (1), (2), (3) and (4) and for comparison purposes the values of $\omega / \omega_{0}$ in modified Lindstedt-Poincare approximation [11] are also given in the tables.

For a nonlinear oscillator with only a quadratic term as a nonlinearity $\left(\alpha_{2} \neq 0\right.$ and $\left.\alpha_{3}=\alpha_{4}=0\right)$, the exact values of frequencies are available in the literature $[29,30]$ and are given in terms of complete elliptic integrals (relation No. (46) of [30]). Mathematica software has been used for calculation of numerical values of elliptic integrals and finding the roots of cubic polynomial equations. For a nonlinear oscillator with mixed parity $\left(\alpha_{2} \neq 0, \alpha_{3} \neq 0, \alpha_{4} \neq 0\right)$, the exact values of frequencies can be calculated [37] by the integral,

$$
\begin{aligned}
& \frac{\omega_{e x}}{\omega_{0}}=2 \pi \\
& \left(\int_{0}^{A} \frac{2 d x}{\sqrt{A^{2}-x^{2}+3 f_{1}\left(A^{3}-x^{3}\right)+4 f_{2}\left(A^{4}-x^{4}\right)+5 f_{3}\left(A^{5}-x^{5}\right)}}+\right)^{-1} \\
& \left.\int_{0}^{B} \frac{2 d x}{\sqrt{B^{2}-x^{2}-3 f_{2}\left(B^{3}-x^{3}\right)+4 f_{2}\left(B^{4}-x^{4}\right)-5 f_{3}\left(B^{5}-x^{5}\right)}}\right)^{-}
\end{aligned}
$$

\begin{tabular}{|c|c|c|c|c|}
\hline$f_{1}$ & $B$ & $\omega_{2 M H P} / \omega_{0}$ & $\omega_{2 M L P} / \omega_{0}$ & $\omega_{\text {exact }} / \omega_{0}$ \\
\hline $\begin{array}{c}0.01 \\
0.05 \\
0.10 \\
0.11 \\
0.12 \\
0.13 \\
0.14 \\
0.15 \\
0.155 \\
0.1565\end{array}$ & $\begin{array}{c}0.722439 \\
0.792221 \\
0.913755 \\
0.947125 \\
0.986189 \\
1.03385 \\
1.09633 \\
1.19283 \\
1.28435 \\
1.3404\end{array}$ & $\begin{array}{c}0.999569 \\
0.987927 \\
0.939194 \\
0.921209 \\
0.898183 \\
0.867497 \\
0.8232207 \\
0.744627 \\
0.649963 \\
0.556534\end{array}$ & $\begin{array}{l}0.999568 \\
0.987913 \\
0.939082 \\
0.921043 \\
0.897928 \\
0.867083 \\
0.822454 \\
0.742729 \\
0.644365 \\
0.527906\end{array}$ & $\begin{array}{l}0.999569 \\
0.987926 \\
0.939193 \\
0.921214 \\
0.898204 \\
0.867561 \\
0.823418 \\
0.745686 \\
0.655595 \\
0.582921\end{array}$ \\
\hline
\end{tabular}

This integral is evaluated numerically by mathematica for $\omega_{\text {exact }} / \omega_{0}$, the exact values of frequencies.

In table 1, it has been assumed that only the hexapole superposition

\begin{tabular}{|c|c|c|c|}
\hline$f_{2}$ & $\omega_{2 M H P} / \omega_{0}$ & $\omega_{2 M L P} / \omega_{0}$ & $\omega_{\text {exact }} / \omega_{0}$ \\
\hline $\begin{array}{l}0.01 \\
0.05 \\
0.10 \\
0.15 \\
0.20 \\
0.25 \\
0.30 \\
0.40 \\
0.50 \\
-0.01 \\
-0.05 \\
-0.10 \\
-0.15 \\
-0.20\end{array}$ & $\begin{array}{c}1.01487 \\
1.072 \\
1.13891 \\
1.20173 \\
1.2612 \\
1.31776 \\
1.37188 \\
1.4739 \\
1.56905 \\
0.984866 \\
0.921355 \\
0.833427 \\
0.730894 \\
0.598426\end{array}$ & $\begin{array}{c}1.01487 \\
1.072 \\
1.1389 \\
1.20173 \\
1.2612 \\
1.31776 \\
1.37188 \\
1.4739 \\
1.56905 \\
0.984866 \\
0.921355 \\
0.833427 \\
0.730894 \\
0.598426\end{array}$ & $\begin{array}{c}1.01487 \\
1.072 \\
1.1389 \\
1.2017 \\
1.2612 \\
1.3177 \\
1.372 \\
1.4739 \\
1.569 \\
0.98487 \\
0.92136 \\
0.83343 \\
0.73099 \\
0.59968\end{array}$ \\
\hline
\end{tabular}
exist and the other multipoles are absent. So, the exact values of secular frequencies $\left(\omega_{\text {exact }} / \omega_{0}\right)$ for different values of $f_{1}\left(f_{2}=f_{3}\right.$ $=0)$ are compared with the results of this paper for second order

Table 1: Comparison of the calculated values of $\omega_{2} / \omega_{0}$ in this paper for only hexapole superposition with the values obtained by modified Lindstedt-Poincare approximation [11] and the exact values.

Table 2: Comparison of the calculated values of $\omega_{2} / \omega_{0}$ in this paper for only octopole superposition with the values obtained by modified Lindstedt-Poincare approximation [11] and the exact values. 
Citation: Doroudi A (2012) Application of a Modified Homotopy Perturbation Method for Calculation of Secular Axial Frequencies in a Nonlinear lon Trap with Hexapole, Octopole and Decapole Superpositions. J Bioanal Biomed 4: 085-091. doi:10.4172/1948-593X.1000068

\begin{tabular}{|c|c|c|c|c|c|}
\hline$f_{1}$ & $f_{2}$ & $B$ & $\omega_{2 M H P} / \omega_{0}$ & $\omega_{2 M L P} / \omega_{0}$ & $\omega_{\text {exact }} / \omega_{0}$ \\
\hline $\begin{array}{l}0.01 \\
0.05 \\
0.10 \\
0.15 \\
0.20 \\
0.25 \\
0.12 \\
0.05 \\
0.07 \\
0.01\end{array}$ & $\begin{array}{l}0.01 \\
0.05 \\
0.10 \\
0.15 \\
0.20 \\
0.25 \\
0.30 \\
-0.05 \\
-0.07 \\
-0.10\end{array}$ & $\begin{array}{l}0.721825 \\
0.775049 \\
0.829421 \\
0.872078 \\
0.905442 \\
0.931747 \\
0.794033 \\
0.824202 \\
0.979204 \\
0.733768\end{array}$ & $\begin{array}{c}1.01478 \\
1.07113 \\
1.13922 \\
1.20686 \\
1.27406 \\
1.34028 \\
1.39885 \\
0.883214 \\
0.698859 \\
0.82468\end{array}$ & $\begin{array}{c}1.01478 \\
1.07113 \\
1.13924 \\
1.20693 \\
1.27422 \\
1.34054 \\
1.39889 \\
0.883152 \\
0.697807 \\
0.824691\end{array}$ & $\begin{array}{c}1.01478 \\
1.07113 \\
1.13922 \\
1.20689 \\
1.27412 \\
1.3404 \\
1.39888 \\
0.883223 \\
0.700873 \\
0.824691\end{array}$ \\
\hline
\end{tabular}

Table 3: Comparison of the calculated values of $\omega_{2} / \omega_{0}$ in this paper for hexapole and octopole superpositions with the values obtained by modified Lindstedt-Poincare approximation [11] and the exact values.

\begin{tabular}{|c|c|c|c|c|c|c|}
\hline$f_{1}$ & $f_{2}$ & $f_{3}$ & $B$ & $\omega_{2 M H P} / \omega_{0}$ & $\omega_{2 M L P} / \omega_{0}$ & $\omega_{\text {exact }} / \omega_{0}$ \\
\hline $\begin{array}{l}0.01 \\
0.02 \\
0.10 \\
0.05 \\
0.07 \\
0.15 \\
0.12 \\
0.14 \\
0.13 \\
0.03 \\
0.05 \\
0.01 \\
0.02\end{array}$ & $\begin{array}{l}0.01 \\
0.02 \\
0.10 \\
0.05 \\
0.08 \\
0.20 \\
0.15 \\
0.30 \\
0.40 \\
-0.05 \\
-0.01 \\
-0.10 \\
-0.08\end{array}$ & $\begin{array}{l}0.01 \\
0.02 \\
0.06 \\
0.03 \\
0.05 \\
0.10 \\
0.04 \\
0.12 \\
0.16 \\
0.02 \\
0.04 \\
0.01 \\
0.03\end{array}$ & $\begin{array}{c}0.73513 \\
0.76452 \\
0.966336 \\
0.823497 \\
0.885144 \\
1.07527 \\
0.894785 \\
0.949597 \\
0.92592 \\
0.825353 \\
0.935453 \\
0.761675 \\
0.89998\end{array}$ & $\begin{array}{c}1.01302 \\
1.02191 \\
1.03587 \\
1.0494 \\
1.06025 \\
1.08029 \\
1.17802 \\
1.31772 \\
1.43909 \\
0.869297 \\
0.844398 \\
0.808686 \\
0.68875\end{array}$ & $\begin{array}{c}1.01302 \\
1.02192 \\
1.03603 \\
1.04941 \\
1.0603 \\
1.0811 \\
1.178 \\
1.31768 \\
1.43654 \\
0.869339 \\
0.844862 \\
0.808704 \\
0.702734\end{array}$ & $\begin{array}{c}1.01302 \\
1.02191 \\
1.03584 \\
1.0494 \\
1.06025 \\
1.08014 \\
1.17802 \\
1.31774 \\
1.43667 \\
0.869302 \\
0.844401 \\
0.808711 \\
0.69135\end{array}$ \\
\hline
\end{tabular}

Table 4: Comparison of the calculated values of $\omega_{2} / \omega_{0}$ in this paper for hexapole, octopole and decapole superpositions with the values obtained by modified LindstedtPoincare approximation [11] and the exact values.

approximation $\left(\omega_{2 M H P} / \omega_{0}\right)$ and the results of modified LindstedtPoincare approximation $\left(\omega_{2 M L P} / \omega_{0}\right)$ obtained in [11].

In table 2, we have considered only the octopole superposition. In this table, the exact values of secular frequencies $\left(\omega_{\text {exact }} / \omega_{0}\right)$ for different values of $f_{2}\left(f_{1}=f_{3}=0\right)$ are compared with the results of this paper for second order approximation $\left(\omega_{2 M H P} / \omega_{0}\right)$ and the results of modified Lindstedt-Poincare approximation $\left(\omega_{2 M L P} / \omega_{0}\right)$ obtained in [11].

In table 3, the hexapole and octopole superpositions are considered and the exact values of secular frequencies $\left(\omega_{\text {exact }} / \omega_{0}\right)$ for different values of $f_{1}$ and $f_{2}\left(f_{3}=0\right)$ are compared with the results of this paper for second order approximation $\left(\omega_{2 M H P} / \omega_{0}\right)$ and the results of modified Lindstedt-Poincare approximation $\left(\omega_{2 M L P} / \omega_{0}\right)$ obtained in [11].

Finally, in table 4, the hexapole, octopole, and decapole superpositions are considered and the exact values of secular frequencies $\left(\omega_{\text {exact }} / \omega_{0}\right)$ for different values of $f_{1}, f_{2}$ and $f_{3}$ are compared with the results of this paper for second order approximation $\left(\omega_{2 M H P} / \omega_{0}\right)$ and the results of modified Lindstedt-Poincare approximation $\left(\omega_{2 M L P} / \omega_{0}\right)$ obtained in [11].

As is seen in the table 1-4, the results of this paper are in excellent agreement with the exact results and are closer to the exact results compared with the results of modified Lindstedt-Poincare approximation obtained in [11].

\section{Conclusion}

In this paper we have derived the equation of ion motion in axial direction of a nonlinear ion trap. The nonlinear ion trap is generated by superposition of weak multipole fields on the pure quadrupole field. Hexapole, octopole, and decapole field superpositions are considered.
The computed axial equation of ion motion is a nonlinear equation with quadratic, cubic and quartic nonlinearity. We have used the modified homotopy perturbation method for solution of the resulted equation and calculation of the axial secular frequencies of the ions in the trap. The results of this paper are compared with the exact results and the results of the modified Lindstedt-Poincare approximation obtained in [11]. There is an excellent agreement between the results of this paper and the exact results.

\section{References}

1. McLachlan NW (1958) Ordinary Non-linear Differential Equations in Engineering and Physical Sciences, Oxford University Press, UK.

2. Franzen J (1993) The non-linear ion trap. Part 4. Mass selective instability scan with multipole superposition. Int J Mass Spectrom Ion Process 125: 165-170.

3. Nappi M, Frankevich V, Soni M, Cooks RG (1998) Characteristics of a broadband Fourier transform ion trap mass spectrometer. Int J Mass Spectrom 177 : 91-104.

4. Sevugarajan S, Menon AG (1999) Field imperfection induced axial secular frequency shifts in nonlinear ion traps. Int J Mass Spectrom 189: 53-61.

5. Sevugarajan S, Menon AG (2000) Frequency perturbation in nonlinear Paul traps:: a simulation study of the effect of geometric aberration, space charge, dipolar excitation and damping on ion secular frequency. Int J Mass Spectrom 197: $263-278$.

6. Sevugarajan S, Menon AG (2001) A simulation study of coupled secular oscillations in nonlinear Paul trap mas spectrometers. Int $\mathrm{J}$ Mass Spectrom 209: 209-226

7. Doroudi A (2009) Calculation of coupled secular oscillation frequencies and axial secular frequency in a nonlinear ion trap by a homotopy method. Phys Rev E Stat Nonlin Soft Matter Phys 80: 056603-56611.

8. Doroudi A (2010) Comparison of calculated axial secular frequencies in nonlinear ion trap by homotopy method with the exact results and the results of Lindstedt-Poincare approximation. Int J Mass Spectrom 296: 43-46. 
Citation: Doroudi A (2012) Application of a Modified Homotopy Perturbation Method for Calculation of Secular Axial Frequencies in a Nonlinear lon Trap with Hexapole, Octopole and Decapole Superpositions. J Bioanal Biomed 4: 085-091. doi:10.4172/1948-593X.1000068

9. Hu H (2006) Solution of a quadratic nonlinear oscillator by the method of harmonic balance. J Sound Vib 293: 462-468.

10. $\mathrm{Hu} \mathrm{H}$ (2007) Solution of a mixed parity nonlinear oscillator: Harmonic balance. J Sound Vib 299: 331-338.

11. Doroudi A, Rezaeian Asl A (2012) Calculation of secular axial frequencies in a nonlinear ion trap with hexapole, octopole and decapole superpositions by a modified Lindstedt-Poincare method. Int J Mass Spectrom 309: 104-108.

12. He JH (2002) Modified Lindstedt-Poincare methods for some strongly nonlinear oscillations: Part I: expansion of a constant. Int J Nonl Mech 37: 309-314.

13. He JH (2002) Modified Lindstedt-Poincare methods for some strongly nonlinear oscillations: Part II: a new transformation. Int J Nonl Mech 37: 315-320.

14. He JH (2006) Some asymptotic methods for strongly nonlinear equations. Int J Mod Phys B 20: 1141.

15. He JH (2004) The homotopy perturbation method for nonlinear oscillators with discontinuities. Appl Math Comput 151: 287-292.

16. Cveticanin L (2006) Homotopy-perturbation method for pure nonlinear differential equation. Chaos, Solitons \& Fractals 30: 1221-1230.

17. Abbasbandy S (2006) Application of He's homotopy perturbation method for Laplace transform. Chaos Solitons \& Fractals 30: 1206-1212.

18. Belendez A, Hernandez A, Belendez T, Neipp C, Marquez A (2007) Application of the homotopy perturbation method to the nonlinear pendulum. Eur $\mathrm{J}$ Phys 28: 93.

19. Belendez A, Hernandez A, Belendez T, Marquez A, Niepp C, et al. (2007) An Improved 'Heuristic' Approximation for the Period of a Nonlinear Pendulum: Linear Analysis of a Classical Nonlinear Problem. Int J Non-linear Sci Numer Simulation 8: 329-334.

20. Ganji DD, Sadighi A (2006) Application of He's Homotopy-perturbation Method to Nonlinear Coupled Systems of Reaction-diffusion Equations. Int J Non-linear Sci Numer Simulation 7: 411-418.

21. He JH (2006) Homotopy perturbation method for solving boundary value problems. Phys Lett A 350: 87-88.

22. Ganji DD (2006) The application of He's homotopy perturbation method to nonlinear equations arising in heat transfer. Phys Lett A 355: 337-341.
23. Chowdhury MSH, Hashim I, Abdulaziz O (2007) Application of homotopyperturbation method to nonlinear population dynamics models. Phys Lett $A$ 368: 251-258.

24. Chowdhury MSH, Hashim I (2007) Solutions of time-dependent Emden-Fowler type equations by homotopy-perturbation method. Phys Lett A 368: 305-313.

25. Dehghan M, Shakeri F (2007) Solution of a partial differential equation subject to temperature overspecification by He's homotopy perturbation method. Phys Scr 75: 778 .

26. Belendez A, Pascual C, Gallego S, Ortuno M, Neipp C (2007) Application of a modified He's homotopy perturbation method to obtain higher-order approximations of an $\times 1 / 3$ force nonlinear oscillator. Phys Lett $A$ 371: 421-426.

27. Belendez A, Pascual C, Ortuno M, Belendez T, Gallego S (2009) Application of a modified He's homotopy perturbation method to obtain higher-order approximations to anonlinear oscillator with discontinuities. Nonlinear Analysis: Real World Applications 10: 601-610.

28. Belendez A, Pascual C, Belendez T, Hernandez A (2009) Solution for an anti-symmetric quadratic nonlinear oscillator by a modified He's homotopy perturbation method. Nonlinear Analysis: Real World Applications 10: 416-427.

29. Apostol BF (2003) On the cubic anharmonic oscillator. J Theoretic Physic 86: 1

30. Amore P, Fernandez FM (2005) Exact and approximate expressions for the period of anharmonic oscillators. Eur J Phys 26: 589.

31. Jackson JD (1999) Classical Electrodynamics, John Wiley \& Sons Inc.

32. Landau LD, Lifshitz EM (1981) Mechanics, Reed Publishing Ltd.

33. Nayfeh AH (1985) Problems in Perturbations. Wiley, New York.

34. Nayfeh AH, Mook DT (1995) Non-linear Oscillations. John Wiley and Sons Inc. New York.

35. Amore P, Aranda A (2005) Improved Lindstedt-Poincaré method for the solution of nonlinear problems. J Sound Vib 283: 1115-1136.

36. Belendez A, Belendez T, Marquez A, Neipp C (2008) Application of He's homotopy perturbation method to conservative truly nonlinear oscillators. Chaos, Solitons \& Fractals 37: 770-780.

37. Leung AYT, Guo Z (2009) Homotopy perturbation for conservative HelmholtzDuffing oscillators. J Sound Vib 325: 287-296. 